MATTEO NACCI

Pontificia Università Lateranense

\title{
DIRITTO CANONICO E DIRITTI DELLE RELIGIONI ${ }^{1}$
}

Sommario: - 1. Rapporto Diritto canonico - Diritti delle religioni: status quaestionis. - 2. La tradizione dell'Institutum Utriusque Iuris della Pontificia Università Lateranense. - 3. Il Decreto Novo Codice e il nuovo "statuto accademico" dello studio dei diritti religiosi in relazione al diritto canonico. - 4. Riflessioni conclusive.

\section{Rapporto Diritto canonico - Diritti delle religioni: status quaestionis}

Nel presentare il rapporto tra il diritto canonico e i diritti delle religioni nella sua dimensione accademica, vorrei partire dallo status quaestionis di questo orientamento di ricerca di alcune Facoltà di Giurisprudenza. Partendo dall'elemento meno positivo, possono essere indicati due interrogativi che gravano sulla solidità di quest'area di studio. Il primo è la pressoché totale disattenzione reale alla conoscenza dei diritti religiosi. In effetti, in un'opera che porta la celeberrima firma dei comparatisti Zweigert e $\mathrm{Kötz}^{2}$, dove i sistemi

\footnotetext{
${ }^{1}$ Conferenza tenuta in occasione della $8^{\circ}$ Giornata Canonistica Interdisciplinare, Diritto canonico tra fondamenti e prassi (a 10 anni da: "Novo Codice"), Pontificia Università Lateranense (Città del Vaticano, 5 marzo 2013).

${ }^{2} \mathrm{Si}$ fa riferimento a K. Zweigert-H. Kötz, Einfürung in die Rechtsvergleichung, 2 voll., Tübingen 1984, edizione italiana a cura di A. Di Majo-A. Gambaro, Introduzione al Diritto Comparato, traduzione di B. Pozzo, vol. I, Principi fondamentali, Milano 1992 e vol. II, Istituti, Milano 1995.
} 
giuridici vengono divisi in sei grandi gruppi ${ }^{3}$, uno di questi - qualificato come "ulteriori sistemi di diritto" - passa rapidamente in considerazione, oltre che il diritto giuridico dell'Estremo Oriente ${ }^{4}$, il cosiddetto sistema dei diritti religiosi nella trattazione delle principali caratteristiche del Diritto islamico e di quello indù ${ }^{5}$ E noto che in quest'opera, pur avendo essa conferito agli studi comparatistici un grande impulso, non si può senz'altro parlare di studio comparato dei diritti religiosi.

Il secondo elemento negativo, che ci riguarda più direttamente, è che l'impulso dato alla materia dello studio del diritto delle religioni in alcune Università di diversi Stati è andato in detrimento dello studio del diritto canonico come ordinamento intrinseco, aggiungendo le considerazioni su quest'ultimo solo a certi aspetti di natura pubblica. In tal senso potrebbe sembrare che dal punto di vista accademico lo studio del diritto delle religioni rappresenti un'ulteriore diminuzione, in centri accademici secolari, della presenza culturale dell'ordinamento canonico. Ovviamente di tutt'altro tenore è il progetto accademico sul diritto comparato delle religioni presente a Lugano in un Centro, però, all'interno di una Facoltà di Teologia ecclesiastica ${ }^{6}$.

\footnotetext{
${ }^{3}$ Tale suddivisione è facilmente riscontrabile anche nell'Indice sommario dell'Opera; cfr. K. ZweIGeRT-H. KöTz, Einfürung in die Rechtsvergleichung, cit., V-VI.

${ }^{4}$ Ivi, 359-372.

${ }^{5}$ Ivi, 372-391.

${ }^{6}$ Faccio riferimento all'Istituto Internazionale di Diritto Canonico e Diritto Comparato delle Religioni, eretto presso la Facoltà di Teologia di Lugano (Svizzera) nell'anno accademico 2001-2002, il quale ha come scopo principlae quello di offrire «la possibilità di una formazione scentifica in Diritto canonico comparato e Diritto ecclesiastico e Diritto comparato delle religioni sulla base di un duplice insegnamento: innanzitutto quello del Concilio Vaticano II, secondo cui "nell'esposizione del Diritto canonico" si deve tener "presente il mistero della Chiesa, secondo la costituzione dogmatica De Ecclesia"; in secondo luogo quello suggerito da Papa Benedetto XVI, ossia la necessità di riscoprire il ruolo del diritto naturale nell'analisi giuridica comparata dei diversi sistemi giuridici religiosi» (IsTITUTo
} 
A questi due elementi negativi, cioè la disattenzione effettiva dei diritti religiosi, da un lato, e il non adeguato equilibrio e comunicazione con il sisitema canonico, dall'altro, fa da contraltare un elemento che invece si deve considerare estremamente positivo e che da solo giustificherebbe lo sforzo per consolidare lo statuto accademico di questo settore di studio. Mi riferisco a quanto uno dei principali studiosi italiani della materia segnala in una delle sue opere: «conoscere le regole a cui si ispirano i comportamenti dei fedeli delle diverse religioni ha oggi un'importanza che supera i confini dell'interesse scientifico e tocca questioni legate alla coesione sociale e alla governance delle diversità» ${ }^{7}$. In questo senso, pur lamentando l'eventuale sacrificio dello studio dell'ordinamento canonico che in qualche occasione ha comportato, credo sia legittimo ritenere globalmente positivo che il sistema dei diritti religiosi abbia interessato, da qualche decennio, molti studiosi del fenomeno giuridico e che oggi si possa parlare di una scienza giuridica che studia i diritti religiosi con metodo comparatistico.

\section{La tradizione dell'Institutum Utriusque Iuris della Pontificia Università Lateranense}

In questo quadro, merita speciale menzione la tradizione delle Facoltà giuridiche dell'Institutum Utriusque Iuris della Pontificia Università Lateranense. Si fa riferimento all'istituzione di un corso

Internazionale di Diritto Canonico e Diritto Comparato delle Religioni, Programma degli Studi, Anno accademico 2011-2012, Lugano 2011, 7).

${ }^{7}$ S. FerRARI (cur.), Introduzione al diritto comparato delle religioni. Ebraismo, islam e induismo, Bologna 2008, prefazione.

${ }^{8} \mathrm{Ci}$ pare molto corretta, a tal proposito, la definizione di Diritto comparato delle religioni di Silvio Ferrari - anche se data in «prima approssimazione» dal suo Autore - di «ramo della scienza giuridica che pone a confronti il diritto prodotto dalle diverse comunità religiose allo scopo di individuarne analogie e differenze» (S. FERRARI (cur.), Introduzione, cit., 37). In tema di diritto comparato delle religioni si veda, ex multis, S. FERRA RI, Lo spirito dei diritti religiosi. Ebraismo, cristianesimo e islam a confronto, Bologna 2002; S. FER RA RI (cur.), Introduzione, cit., in particolare il capitolo dedicato agli studi - italiani ed europei - di diritto comparato delle religioni, 23-37, ed ivi ampia e qualificata bibliografia. 
di Diritto islamico, tenuto per molto tempo da professori originari del Libano (ad esempio, il prof. El-Hachem) ${ }^{9}$, la cui vicinanza con la cultura giuridica musulmana è ben nota. Si è trattato di un periodo in cui sulle riflessioni sistematiche è prevalso il valore della conoscenza reale e dell'esperienza vissuta, di cui gli stessi docenti potevano rendere testimonianza ben accreditata. Nell'anno accademico 1985-1986 $\mathrm{fu}$ anche istituito il corso di Diritto ebraico, tenuto da una docente (la prof.ssa Daniela Piattelli) conoscitrice del diritto romano e dell'antico Oriente mediterraneo e con un programma che cominciava a porre le basi di quella che più tardi sarebbe stata la comparazione degli ordinamenti giuridici religiosi ${ }^{10}$. Ma si trattava di basi ancora molto limitate e nonostante gli aspetti positivi si era ben lontani da una comparazione sistematica con il diritto canonico.

Lo sviluppo che nel corso degli anni ha avuto il diritto comparato, nella Facoltà di Diritto Canonico, come una delle due dimensioni (insieme a quella internazionale) in cui da sempre il nostro Ateneo ha ritenuto di dover tradurre quanto previsto dall'articolo 56 delle Ordinationes ${ }^{11}$ della Costituzione Apostolica Sapienta

\footnotetext{
${ }^{9}$ Il Prof. Mounged El-Hachem tenne il corso di Ius Islamicum, sostituendo il Prof. Hitti, a partire dall'anno accademico 1991-1992; cfr. Pontificia Universitas Later Anensis, Ordo anni academici 1991-1992, Città del Vaticano 1991, 194-195.

${ }^{10}$ Cfr. Pontificia Universitas Lateranensis, Ordo anni academici 1985-1986, Città del Vaticano 1985, 205-206.

${ }^{11}$ Sacra Congregatio pro Institutione Catholica, Ordinationes ad Constitutionem Apostolicam «Sapientia Christiana» rite exsequendam, 29 aprilis 1979, art. 56, AAS 71(1979), 514-515: «Disciplinae obligatoriae sunt: $1^{\circ}$ in primo cyclo: a) Institutiones generales Iuris Canonici; b) Elementa Sacrae Theologiae (praesertim ex Ecclesiologia et Theologia sacramentaria) et Philosophiae (praesertim ex Ethica et Iure naturali), quae suapte natura requirantur ante studium Iuris Canonici; quibus utiliter addi possunt elementa ex scientiis anthropologicis cum scientia iuridica conexis. $2^{\circ}$ in secundo cyclo:a) Codex Iuris Canonici secundum omnes eius partes, aliaeque leges canonicae; b) Disciplinae conexae, cuius modi sunt: Philosophia Iuris, Ius publicum ecclesiasticum, Institutiones Iuris Romani, Elementa Iuris Civilis, Historia Iuris Canonici, cum propriis exercitationibus et seminariis, quadam quoque commentatione conscripta. $3^{\circ}$ in tertio cyclo:Determinent Statuta Facultatis, quae
} 
Christiana $^{12}$ - e ribadito nel Decreto Novo Codice $^{13}$ - sul bisogno che negli studi canonistici non manchi l'approfondimento degli elementa iuris civilis, ha accresciuto l'attenzione nei confronti dei diritti religiosi, seppur solo indirettamente, in quanto alcuni dei grandi sisitemi giuridici hanno un contesto culturale inseparabile dalla rispettiva matrice religiosa. Si tratta di una tappa che pone le basi per sviluppare e riempire quella lacuna precedentemente indicata, cioè la metodologia della comparazione pur ancora molto incipiente nelle sue ricadute sui diritti religiosi.

\section{Il Decreto Novo Codice e il nuovo "statuto accademico" dello studio dei diritti religiosi in relazione al diritto canonico}

Una tappa successiva credo che possa essere individuata nella ricorrenza che in questa Giornata stiamo celebrando, cioè il Decreto

disciplinae speciales et exercitationes atque seminaria praescribenda sint secundum propriam Facultatis naturam et peculiares studentium necessitates».

${ }^{12}$ Ioannes Paulus Pp II, Const. Ap. de studiorum Universitatibus et Facultatibus Ecclesiasticis: Sapientia christiana, 15 aprilis 1979, AAS 71(1979), 469-499.

${ }^{13}$ Congregatio de Institutione Catholica, Decretum quo ordo studiorum in Facultatibus Iuris Canonici innovatur: Novo Codice, 2 septembris 2002, art. 56, A AS 95(2003), 281-285, in part. 283-284: «Disciplinae obligatoriae sunt: $1^{\circ}$ in primo cyclo: a) elementa philosophiae; anthropologia philosophica; metaphysica; ethica; b) elementa theologiae: introductio in S. Scripturam; theologia fundamentalis: divina revelatio, eiusdemque transmissio et credibilitas; theologia trinitaria; christologia; tractatus de gratia; peculiari autem modo ecclesiologia, theologia sacramentalis generalis et specialis, theologia moralis fundamentalis et specialis; $c$ ) institutiones generales iuris canonici; d) lingua latina. $2^{\circ}$ in secundo cyclo: a) Codex Iuris Canonici vel Codex Canonum Ecclesiarum Orientalium secundum omnes eorundem partes aliaeque leges canonicae; b) disciplinae conexae: theologia iuris canonici; philosophia iuris; institutiones iuris romani; elementa iuris civilis; historia institutionum canonicarum; historia fontium iuris canonici; relationes Ecclesiam inter et societatem civilem; praxis canonica administrativa et iudicialis; c) introductio in Codicem Canonum Ecclesiarum Orientalium pro studentibus Facultatis Iuris Canonici latini; introductio in Codicem Iuris Canonici pro studentibus Facultatis Iuris Canonici orientalis; d) lingua latina; e) disciplinae speciales et exercitationes atque seminaria ab unaquaque Facultate praescripta. $3^{\circ}$ in tertio cyclo: a) latinitas canonica; b) cursus speciales vel exercitationes ab unaquaque Facultate praescripta». 
Novo Codice sulla riforma degli studi canonistici e, di conseguenza, degli studi in utroque iure. In effetti, come si legge nel Proemio del Decreto e si evince dal titolo che abbiamo dato a questa giornata, la riforma muove dall'obiettivo di valorizzare il fondamento teologico del diritto canonico ${ }^{14}$. Nel contesto della comparazione che contraddistingue la nostra Accademia, detto obiettivo ha indotto a riscoprire l'importanza dei sistemi giuridici religiosi in quanto diritti anch'essi teologicamente fondati. Questa riscoperta è iniziata a tradursi in un perfezionamento del Programma degli Studi dove l'attenzione ai diritti religiosi possa risultare più solida e sistematica. In tal senso, un primo passo è stato fatto dando più spazio anche agli ordinamenti giuridici religiosi nel programma del corso "Sistemi di diritto civile comparato"15. Ma il passo verso il quale ci si incammina, in un futuro non lontano, è che vi sia una materia autonoma che mantenendo intatto lo studio separato di ogni sistema affronti direttamente la comparazione tra i medesimi ed includa i grandi temi della loro relazione con il diritto canonico.

Riferiamoci ora a quali possano essere le ragioni ultime di questo non lontano statuto accademico autonomo nello studio dei diritti religiosi in relazione al diritto canonico. Credo che le ragioni possano essere ricondotte a due gruppi di motivazioni. Il primo è ancora di natura teologica. In effetti, lo studio dei sistemi giuridici religiosi è

\footnotetext{
${ }^{14}$ La valorizzazione del fondamento teologico del Diritto canonico si comprende chiaramente laddove il Decreto del 2002 con cui è stato rinnovato l'ordine degli studi nelle Facoltà di Diritto canonico auspicava che i Codici di diritto canonico latino ed orientale - fossero esposti «sub luce Ecclesiologiae Vaticani II, cuius notae huc spectantes in Constitutionibus Apostolicis Ioannis Pauli II Sacroe disciplinai leges et Sacri Canones in synthesim redactae indicantur. Hic autem novus prospectus theologicus quo in iure canonico exponendo intenditur, ulterius tempus exigit quam limites biennii permittunt. Accedit quod praeter studium linguae latinae aliae disciplinae auxiliares et cursus optionales additi sunt qui necessarii hodie videntur ad formationem institutionalem in iure canonico complendam» (CONGREGATIO DE Institutione Catholica, Decretum quo ordo studiorum in Facultatibus Iuris Canonici innovatur: Novo Codice, cit., 282).

${ }^{15}$ Cfr. Pontificia Universitas Lateranensis, Ordo anni academici 2012-2013, Città del Vaticano 2012, 381-382.
} 
la via più adeguata per coinvolgere gli studi giuridici nella grande sfida che oggi rappresenta il dialogo interreligioso ed il dialogo ecumenico $^{16}$; su quest'ultimo aspetto non mi soffermo in quanto nel sistema canonico una specialissima sfida ecumenica grava sulle Chiese orientali alla cui organizzazione giuridica, nel nostro Piano di Studi, viene riservato un grande spazio con tre materie obbligatorie ed un corso opzionale ${ }^{17}$.

Il secondo gruppo di ragioni è di natura sociologica. Mi riferisco al fenomeno della globalizzazione e dell'immigrazione, che non si esaurisce nella sua dimensione meramente sociologica ma che sprona verso ciò che è autorevolmente denominata "globalizzazione giuridica"18. A questa impegnativa sfida, nello studio dei diritti reli-

\footnotetext{
${ }^{16}$ Sul punto si consenta il rinvio a M. NACCI, Le consuetudini religiose: un contributo della storia del diritto in un contesto di libertà religiosa e pluralismo culturale, Apollinaris LXXXIII (2010), 551-570.

${ }^{17} \mathrm{Si}$ fa riferimento, per l'Anno Accademico 2012-2013, ai corsi obbligatori "Diritto Comune delle Chiese Orientali I", "Diritto Comune delle Chiese Orientali II", "CIC e CCEO comparati" e al corso opzionale "L'applicazione della pena. Comparazione tre CCEO e CIC 83"; cfr. Pontificia Universitas Lateranensis, Ordo anni academici 2012-2013, cit., 354-355, 375, 384.

${ }^{18}$ Sugli effetti della globalizzazione nell'ambito delle religioni si veda, H. KüNG, Scontro di civiltà ed etica globale: globalizzazione, religioni, valori universali, pace, Roma 2005; R. PAPINI, Globalizzazione: culture e religioni, Roma 2005; V. Roldán, Valori, cultura e religioni: processi di globalizzazione e mutamenti sociali, Milano 2011; B. SALVARANI, Il fattore R: le religioni alla prova della globalizzazione, Bologna 2012; S. SAngalli-A.Piccinin, Fedi e globalizzazione cercando valori condivisi, Soveria Mannelli (CZ) 2013. Sulla globalizzazione giuridica e sugli effetti dal punto di vista sociologico si veda, ex multis, M. R. Ferrarese, Le istituzioni della globalizzazione - Diritto e diritti nella società transnazionale, Bologna 2000; M. R. Ferrarese, Diritto sconfinato: inventiva giuridica e spazi nel mondo globale, Roma 2006; F. GALGANO, I caratteri della giuridicità nell'era della globalizzazione, Sociologia del Diritto XXX(2003), 7-17; S. MarotTA, Le nuove feudalità: società e diritto nell'epoca della globalizzazione, Napoli 2007; S. SAssen, Una sociologia della globalizzazione, Torino 2008; G. TAssinari, Globalizzazione, Milano 2009; V. Cotesta, Sociologia del mondo globale, Roma-Bari 2010; M. NACCI, Decidere 'per ragioni': l'arbitrato. Profili storico-giuridici, Apollinaris LXXXIV(2011), 311-333, in part. 327-329; B. Andò-F. VeCCHIO, Costituzione, globalizzazione e tradizione
} 
giosi è lecito aspettarsi dal diritto canonico e dalla loro sistematica comparazione un contributo molto importante per l'attuale fase della storia: quello cioè di orientare assiologicamente in senso positivo la globalizzazione e il corrispettivo pluralismo culturale che la contraddistingue $^{19}$. Senza detto orientamento assiologico, la connessione tra le culture giuridiche potrebbe esaurirsi in mero multiculturalismo infruttuoso, anzi, non esente dal rischio di prevaricazione culturale, concetto con il quale si esprime l'indebito predominio di una cultura sulle altre, tanto nel pensiero quanto nelle legislazioni e soprattutto nei comportamenti $\mathrm{i}^{20}$. Molte ragioni antropologiche possono addursi sui limiti di questo orientamento; la più chiara è che tra tutti i fenomeni umani quello religioso è il meno adatto per essere affrontato giuridicamente in termini di prevaricazione culturale, in quanto radicato nelle più intime convinzioni personali e collettive ${ }^{21}$.

L'orientamento assiologico cui può contribuire il diritto canonico nella comparazione con gli altri diritti religiosi favorirebbe, se non altro, maggiori livelli della cosiddetta interculturalità, intesa come abbandono della prevaricazione in vista della creazione di rapporti orizzontali tra le diverse culture. L'interculturalità poggia sul presupposto consistente nell'accettazione del valore 'relativo' della propria cultura su una questione pratica o su un tema specifico. Certamente la méta più ambita nello studio del diritto canonico con i diritti religiosi sarebbe quella di superare la mera interculturalità per favorire il dialogo interculturale, un obiettivo con il quale si evidenzia non solo il fondamentale ed ineliminabile rispetto per le culture diverse dalla propria, ma anche la volontà e la capacità di accoglierne i contenuti ${ }^{22}$.

In quest'ottica del dialogo interculturale, una prima area di studio sugli aspetti giuridici coinvolti nello studio del dirtitto canonico

giuridica europea, Padova 2012; Z. BAuman, Dentro la globalizzazione: le conseguenze sulle persone, Roma 2012.

${ }^{19}$ Sul concetto di pluralismo culturale si rinvia a M. NACCI, Le consuetudini religiose, cit., 553-554.

${ }^{20}$ Ibidem.

${ }^{21}$ Ibidem.

${ }^{22}$ Ibidem. 
e dei diritti religiosi è quella dei rapporti giuridici intersoggettivi tra persone appartenenti a diverse religioni e, in ogni caso, rispetto a quelle materie dove si può ritenere aspirazione comune e speciale sensibilità condivisa dai vari diritti religiosi la difesa della libertà e della dignità umana. Penso, per esempio, all'istituzione familiare e dentro di essa specialmente all'istituzione matrimoniale; penso anche all'educazione, alla difesa della vita, alla giustizia sociale eccetera. Lo sviluppo dello studio delle legislazioni religiose su queste materie può favorire in ogni caso la conoscenza reciproca degli altri e la stima verso le differenze ${ }^{23}$.

È ovvio che lo spazio potenzialmente più adatto e pieno di ricchezza nello studio del diritto comparato delle religioni e del diritto canonico sembra essere quello del diritto pubblico. Concretamente quello del progresso rispetto agli orientamenti legislativi secolari in materia di libertà religiosa. Basti pensare ai risultati positivi cui porta, per esempio, lo studio di una fonte del diritto così rilevante per i sistemi giuridici qual'è la consuetudine. La comparazione dell' istituto della consuetudine nelle tre grandi tradizioni religiose (canonica, ebraica, musulmana) può offrire come principale risultato la stima e la comprensione del fattore religioso come elemento di coesione positiva di una comunità in quanto identificata in un sistema di valori ${ }^{24}$.

Certamente, in un contesto di sempre più accentuata coesistenza di molteplici tradizioni religiose, tale elemento positivo non è ancora

\footnotetext{
${ }^{23}$ In tema di 'stima delle differenze', inteso come modus procedendi per l'attuazione pratica non solo della interculturalità ma del dialogo interculturale, particolarmente interessante è stato un Convegno celebratosi nella Facultad de Derecho dell'Universidad de Navarra (Pamplona-Spagna) dal titolo "Los límites de la diferencia: la protección de la diversidad cultural y religiosa en el derecho contemporáneo", nel quale insieme a studiosi di provenienza internazionale è stato possibile con una conferenza dal titolo Libertà religiosa e pluralismo culturale nell'analisi delle consuetudini religiose secondo la prospettiva della storia del diritto.

${ }^{24}$ Per un primo studio sulla comparazione del fenomeno consuetudinario nel diritto canonico, musulmano ed ebraico si consenta il rinvio a M. NACCI, Canonica consuetudo, urf, minhag. Profili di comparazione, Tesi di dottorato di Ricerca, Corso di Dottorato di Ricerca in Filosofia del Diritto, curriculum: Discipline canonistiche ed ecclesiasticistiche, ciclo XXII, Milano 2010.
} 
sufficiente per illuminare gli indirizzi legislativi in materia di libertà religiosa. Detto elemento deve essere confrontato con il bisogno di assicurare armonia con l'ordine pubblico. Ciò sembra suggerire orientamenti legislativi improntati alla mera tolleranza e coesistenza di ciascuna identità religiosa, trasformando la cautela sull'ordine pubblico in mera limitazione.

In realtà, il fenomeno consuetudinario non rivela solo l'osservanza di tradizione, ma anche e soprattutto la capacità di produrla dal basso, con forza normativa, come risposta alle necessità derivanti dai rapporti intersoggettivi religiosamente motivati. In tal senso, nell'affrontare giuridicamente i nuovi rapporti che caratterizzano l'era della globalizzazione nella quale viviamo, le tradizioni religiose possono risultare fattore motivante insostituibile nell'assicurare un'identità civica dove il pluralismo culturale e religioso riesca a dispiegarsi nella sua forma più nobile, cioè come dialogo interculturale, evitando ogni forma di isolamento con gli annessi pericoli che ciò comporterebbe per la società.

\section{Riflessioni conclusive}

Credo che possa essere interessante per ulteriori spunti di riflessione in materia, il concetto di "identità civica multipla" che qualche autore applica concretamente all'identità del cittadino europeo ${ }^{25}$. Con tale concetto si fa riferimento ad un'insufficienza, ad un modello regolatore e ad un minimo non negoziabile. L'insufficienza è data dall'assumere come prospettiva la mera integrazione tra culture, ricondotta spesso al solo apprendimento della lingua del Paese di accoglienza. Il modello è quello della laicità positiva come principio istituzionale regolatore del pluralismo religioso, che si fonda nella stima di ogni tradizione religiosa, come elemento costruttivo del bene comune, attesa la proiezione universale dei loro valori portanti. Il minimo è dato dalla dignità della persona umana, valore non negoziabile

\footnotetext{
${ }^{25}$ Cfr. L. Gerosa, L'identità laica dei cittadini europei: inconciliabile con il monismo islamico? Implicazioni giuridico-istituzionali del dialogo interreligioso, Soveria Mannelli (CZ) 2009, 45-73.
} 
e criterio discriminante nel fissare eventuali collisioni tra le singole tradizioni religiose e l'ordine pubblico ${ }^{26}$.

Dal punto di vista etico, infine, il supporto del programma delineato risiede nella prospettiva che esprime il concetto di dialogo interculturale; sul punto che ho analizzato, tale dialogo ha due principali implicazioni: 1) assicurare la presenza efficace delle varie culture religiose nella sfera pubblica; 2) evitare ogni uguaglianza neutralizzante valorizzando la ricchezza delle differenze caratterizzanti ciascuna tradizione. La stima delle differenze non deve costituire ostacolo per il reciproco riconoscimento di principi fondamentali comuni, che oggi sono facilmente rapportabili ai diritti umani.

Allo stesso tempo, poiché la religione è radicata nella libertà del singolo, lo studio del diritto canonico e dei diritti delle religioni con metodo comparatistico potrà essere annoverato tra quelle iniziative volte a favorire che ogni persona possa rendere più autentica la propria opzione di senso sulla vita facilitando, così, uno spirito critico ed autocritico sulla propria tradizione religiosa in ciò che possa avere di obsoleto e meno adatto e proponendo anche nuove vie di condivisione che potranno caratterizzare forme di solidarietà più efficaci e congeniali alle esigenze delle nuove società multiculturali per mezzo delle quali «quo namque plenius res orbemque cognovit homo, eo magis ipsemet cognoscit se unica in sua natura» ${ }^{27}$.

\section{Canon law and Laws of religions}

This work is a conference in the Institutum Utriusque Iuris of the Pontifical Lateran University that want to consider the report of Canon law and Laws of religions in the academic and scientific viewpoint. In this perspective, I focused upon the tradition of Pontifical Lateran University about Laws of religions studies and the important "message" of Novo Codice

\footnotetext{
${ }^{26}$ Ibidem.

${ }^{27}$ IOANNES PAUlus PP II, Litterae Encyclicae cunctis catholicae Ecclesiae episcopis de necessitudinis natura inter utramque: Fides et Ratio, 14 septembris 1998, Prooemium, n. 1, AAS 91(1999), 5.
} 
Decree in order to a new "academic statute" of the Laws of religions studies, in relation to Canon law, in our multicultural societies of the third millennium.

Parole Chiave: diritto canonico; Pontificia Università Lateranense; diritti delle religioni; Decreto Novo Codice; società multiculturali

Key Words: canon law; Pontifical Lateran University; laws of religions; Novo Codice Decree; multicultural societies

Nota o Autorze:

Prof. Matteo Nacci - is full professor of History of Law and director of Department of History of Law in the Pontifical Lateran University. 